\title{
REHABILITATION AFTER PARTIAL ARTROSCOPIC MENISCECTOMY
}

Maja Manoleva, Nikolikj Dimitrova E., Kalcovska Ivanovska B., Gocevska M., Malinovska Nikolovska L., Koevska V., Mitrevska B., Gerakaroska Savevska C.

\section{Introduction}

Adequate rehabilitation after partial artroscopic meniscectomy

has important role in good functional status of the patients.

\section{Purpose}

To evaluate the effect of the rehabilitation in restoring functional ability of the knee.

\section{Material and methods}

In this retrospective study 20 patients with partial artroscopic meniscetomy were included. This study was performed from January to December 2016. All patients were referred for outpatient rehabilitation. Every patient received electrotherapy (dyadinamic current, interferential currents), cryotherapy, exercises

by the protocol after

meniscectomy and aquatic exercises in a swimming pool during 3 weeks. The effects of therapy were evaluated by measurement of range of motion in the knee and tight circumference before and after the 3 weeks of rehabilitation.

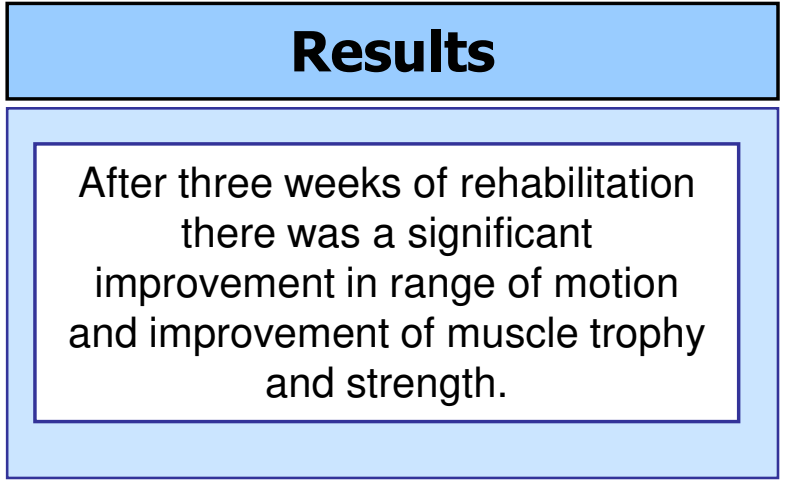

Flexion of knee before and after rehabilitation
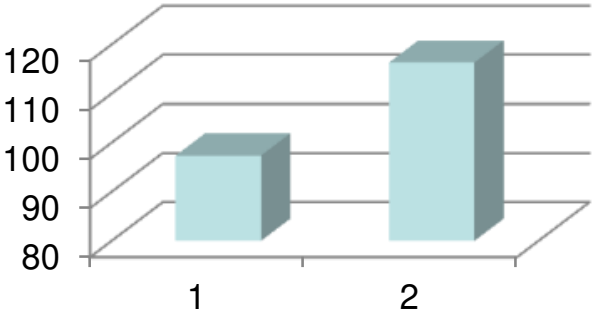

Extension of knee before and after rehabilitation

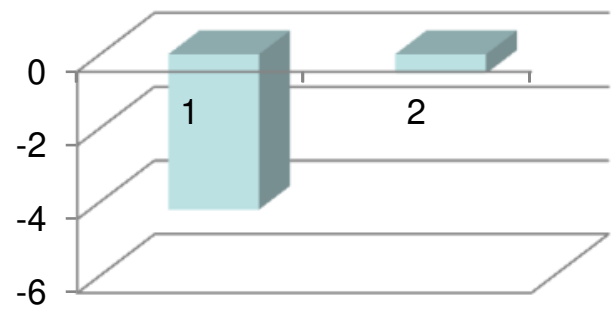

\section{Conclusion}

Rehabilitation with electrotherapy, exercises and hydrotherapy has important role and positive effect of the functional status of the patient after partial artroscopic meniscetomy. 